\title{
Reversibly Thermoswitchable Two-Dimensional Periodic Gratings Prepared from Tethered Poly( $N$-isopropylacrylamide) on Silicon Surfaces
}

\author{
Jem-Kun Chen, ${ }^{* \dagger}{ }^{\dagger}$ Jing-Hong Wang, ${ }^{\dagger}$ Chih-Chia Cheng, ${ }^{\ddagger}$ and Jia-Yaw Chang ${ }^{\S}$ \\ ${ }^{\dagger}$ Department of Materials Science and Engineering and ${ }^{\S}$ Department of Chemical Engineering, National Taiwan University of Science \\ and Technology, 43, Sec. 4, Keelung Road, Taipei, 106, Taiwan, Republic of China \\ ${ }^{\ddagger}$ Department of Applied Chemistry, National Chiao Tung University, 1001 University Road, Hsinchu, Taiwan 300, Republic of China
}

ABSTRACT: In this study we used atom transfer radical polymerization to graft poly $(\mathrm{N}$-isopropylacrylamide) (PNIPAAm) onto flat Si substrates. We then applied very largescale integration and reactive ion etching sequentially to generate 200-nm-scale hole arrays of tethered PNIPAAm as two-dimensional periodic concave gratings (2DPCGs) on the $\mathrm{Si}$ surfaces. The hole array structures of tethered PNIPAAm could be created and erased reversibly at 25 and $40{ }^{\circ} \mathrm{C}$,

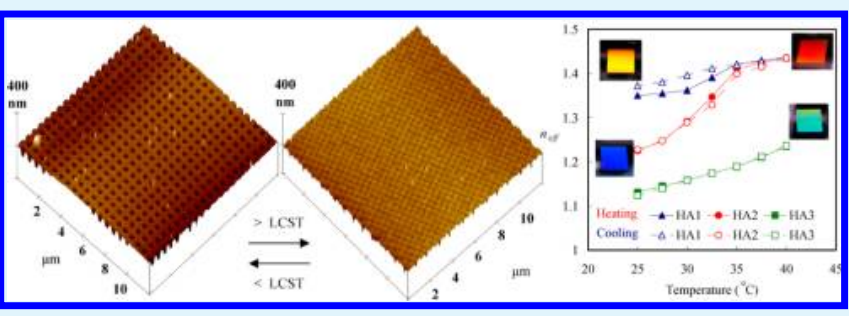
respectively, leading to significant changes in the effective refractive index $\left(n_{\text {eff }}\right)$. The values of $n_{\text {eff }}$ of the 2DPCGs were related to the depth of their holes generated after etching for various times, resulting in a color change from blue to red that could be observed by the naked eye at incident angles of $10-20^{\circ}$. Moreover, we used effective-medium theory to calculate the filling factors of air inside the 2DPCGs to verify the structural changes of the tethered PNIPAAm. Such designed 2DPCGs of thermorespective hydrogel films have potential applications in temperature-responsive optical devices [e.g., as antireflection structured surfaces (ARSs)] that operate at both visible and nearinfrared wavelengths.

KEYWORDS: PNIPAAm brush, two-dimensional periodic grating, refractive index

\section{INTRODUCTION}

Subwavelength structured (SWS) surfaces are attractive components for new optical elements, and many different elements with these surfaces have been developed. Subwavelength structures exhibit artificial refractive indices, from birefringence, and resonance and band gap effects. Their mean refractive indices can be controlled by the filling factors of the structures, allowing a desired distribution of refractive indices can be realized; their nonsymmetric structures cause optical anisotropy, so-called form birefringence. Moreover, when the grating period is on the order of the wavelength of light, the light wave may resonate and become reflected within the structure, so that resonant reflection occurs. Together, these optical features can be used to produce new optical elements. Subwavelength elements were first proposed in the beginning of the $1980 \mathrm{~s}^{1}{ }^{1} 20$ years later, nanofabrication technologies have become paramount for the fabrication of SWS surfaces with applications ranging from microelectronics, ${ }^{2}$ biosensors, ${ }^{3}$ solar cells, ${ }^{4}$ data storage, ${ }^{5}$ and micro-optical components. ${ }^{6}$ The process of patterning, commonly referred to as lithography, involves a flow of information that typically begins with the design of a pattern in terms of a data set and ends with a patterned array of features on the surface of a substrate.

When a light wave is applied incident to a substrate, Fresnel reflection can occur at the material's surface; an SWS can suppress this behavior. A microstructured surface that provides an antireflection effect is known as a "motheye"; the structured surface is called an antireflection structured surface (ARS). ${ }^{7}$ The ARS consists of a pyramidal or conical subwavelength microstructure; Figure 1 presents a schematic representation of porous surface of an ARS. The effective refractive index increases gradually from that of air to that of the substrate; this

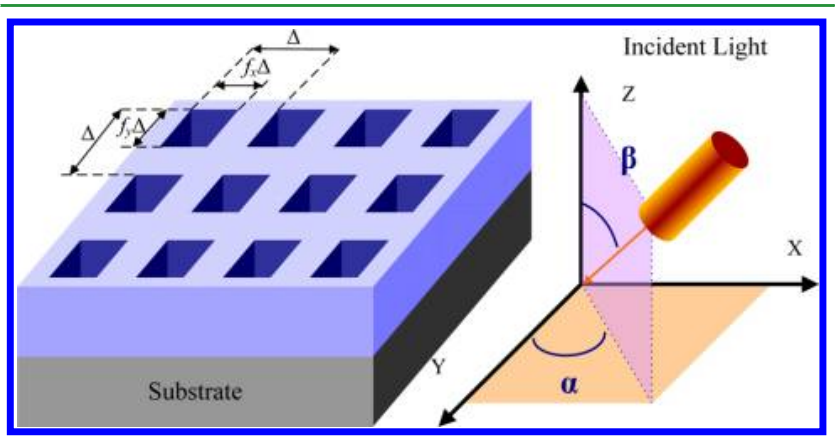

Figure 1. (a) Symmetric two-dimensional periodic concave grating (2DPCG) having filling factors of air in the $x$ and $y$ directions of $f_{x}$ and $f_{y}$, respectively. (b) Schematic representation of light traveling with incident angles $\alpha$ and $\beta$ through a porous surface.

Received: August 28, 2012

Accepted: March 27, 2013

Published: March 27, 2013 
gradual change decreases the reflection of light at the substrate surface. ${ }^{8,9}$ The ARS is formed from the material of the substrate, increasing the performance demands of optical components fabricated from glass-based optical materials. In addition, the mean refractive index of such a structure is distributed vertically from that of the air to that of the substrate. When the mean refractive index changes laterally, the elements may act to form a highly efficient diffraction grating. ${ }^{10}$ Early approaches with glass employed phase separation and etching processes to create porous surfaces; more-recent approaches have employed sol-gel processes ${ }^{11}$ and the adsorption of colloids onto polyelectrolyte multilayers, ${ }^{12}$ among others. Nanoporous polymer coatings created through the selective dissolution of spin-coated films has also been explored recently. ${ }^{13}$

The use of polymers as building blocks for surface modification allows the preparation of "smart" or responsive surfaces based on conformational changes in the polymer backbones. ${ }^{14}$ Stimuli-responsive hydrogels, which undergo large reversible volume changes upon variations in environmental factors, are excellent candidate materials for chemical and biological detection applications. ${ }^{15,16}$ The synthesis and controlled modulation of engineered surface patterns has been applied to observe the diffraction patterns of environmentally responsive hydrogels. ${ }^{17}$ When a grating interacts specifically or nonspecifically with target molecules, its geometrical parameters and/or refractive index contrast will typically change. ${ }^{18}$ Through improvements in nanofabrication technologies, the surface patterns of hydrogels can approach SWS surfaces exhibiting high stability and high chromatic aberration. Nevertheless, the stimuli-responsiveness of surface patterns of hydrogels formed without tethering the chain end to the substrate might not undergo complete reversal after several cycles of use. In previous studies, we grafted polymer chains as surface-tethered patterns, possessing high duty and aspect ratios, onto silicon surfaces. ${ }^{19,20}$ Herein, we describe a simple method, using lithography and reactive ion etching (RIE) techniques, for patterning poly $(\mathrm{N}$-isopropylacrylamide) (PNIPAAm) brushes as two-dimensional periodic concave gratings (2DPCGs), nanoporous analogues. For one-dimensional periodic relief gratings (1DPRGs), the effective refractive indices for transverse magnetic (TM) and transverse electric (TE) polarization are perpendicular and parallel to the linepatterns, respectively. In theory, a $1 D P R G$ can display solely one pure color, visible to the naked eye, along the TM and TE directions. Nevertheless, it is very difficult for the naked eye to observe along the TM and TE directions precisely; therefore, 1DPRGs always present composed color images, because their colors can vary sensitively with the angles $\alpha$ and $\beta$ (Figure 1). ${ }^{6,18,20}$ Unlike 1DPRGs, the colors of 2DPCGs, observed by the naked eye, are dependent solely on the angle $\beta$, thereby providing better stability and chromatic aberration regardless of the visual angle. In addition, such hydrogels coated in patterns on the substrate without substantial tethering to the surface might not undergo complete reversal after several cycles of use because the hydrogel might dissolve into the aqueous solution. Therefore, in this study we have fabricated 2DPCGs featuring covalently tethered PNIPAAm for use as thermoresponsive nanoporous surfaces-that is, as transition media for thermal detection. We have also demonstrated that thin films graded in refractive index $(n)$ are readily obtainable using this approach. Under specific conditions, the transformation to the 2DPCG structure can be completely reversed, making it possible to create reversible 2DPCG thin films. Specifically, we have utilized thermally induced reformation in a PNIPAAm grating system to enable large-scale morphological reorganization, resulting in variations in refractive index. This versatile process is particularly amenable to the creation of large-area uniform coatings on essentially any surface with precise control over thickness and optical properties. ${ }^{21}$ In addition, it is now possible to create 2DPCG thin films that are ideally suited for switchable antireflection applications by cycling between a porous and nonporous state.

\section{EXPERIMENTAL SECTION}

Materials. Single-crystal Si wafers, $\mathrm{Si}(100)$, polished on one side (diameter 6 in.) were supplied by Hitachi (Japan) and cut into $1.5 \mathrm{~cm}$ $\times 1.5 \mathrm{~cm}$ samples. The materials used for graft polymerization-3aminopropyltriethoxysilane (AS), 2-bromo-2-methylpropionyl bromide (BB), $N$-isopropylacrylamide (NIPAAm), copper(I) bromide, copper(II) bromide, triethylamine (TA), and 1,1,4,7,7-pentamethyldiethylenetriamine (PMDETA) - were purchased from Acros Organics. NIPAAm, PMDETA, AS, and BB were purified through vacuum distillation prior to use. All other chemicals and solvents were of reagent grade and purchased from Aldrich Chemical.

Patterning the Nanoporous PNIPAAm Surface. The basic strategy for the fabrication of the patterned nanoporous polymer brushes using the very large-scale integration (VLSI) process was developed in a previous study. ${ }^{22}$ The process is depicted in Figure 2. (A) To immobilize the ATRP initiator, the Si substrate was immersed in AS in toluene. Sequentially, the sample was immersed in a solution of $\mathrm{BB}$ and $\mathrm{TA}$ in tetrahydrofuran (THF). For the preparation of PNIPAAm brushes on the Si-AS-BB surface, NIPAAm, $\mathrm{Cu}(\mathrm{I}) \mathrm{Br}$, $\mathrm{CuBr}_{2}$, and PMDETA were added to extra-dry dimethylformamide (DMF). After polymerization, the wafer was placed in a Soxhlet apparatus to remove any unreacted monomer, catalyst, and nongrafted material. In addition, the tethered PNIPAAm brushes were stripped from the surface for analysis using gel permeation chromatography (GPC). Monodisperse polymer standards (Polymer Lab, Agilent) were used to generate a calibration curve. (B) E-beam lithography was then used to pattern the photoresist as 2DPCG, which, after development, had a resolution of $200 \mathrm{~nm}$. (C) Inductively coupled plasma/reactive ion etching (ICP-RIE; Inductive Couple Plasma Etcher, ICP Etcher) was used for dry etching after photoresist had been patterned onto the surface to cover the PNIPAAm brushes as a protection mask. Only the exposed PNIPAAm brushes were dry etched and treated through RIE. The plasma inside was generated and accelerated by the generator power and platen power, respectively. Another process parameter controlled for this study was the mixture of gases and their ratios. ${ }^{23}$ These conditions have generally been used for deep dry etching without any special surface requirements on solid state substrates. (D) Finally, the remaining photoresist hard mask was removed from the PNIPAAm surface through washing with solvents prior to further surface measurements, leaving behind a 2DPCG of tethered PNIPAAm.

Characterization of Thermally Responsive 2DPCG of Tethered PNIPAAm. Hole arrays (2DPCGs) of the PNIPAAmgrafted surfaces were analyzed using ellipsometry (SOPRA SE-5, France) and X-ray photoelectron spectroscopy (XPS; Scientific Theta Probe, UK). The morphologies of various patterns of PNIPAAm brushes were analyzed using atomic force microscopy (AFM; Veeco Dimension 5000 scanning probe microscope). Both thermal treatment and treatment with a poor solvent could lead to phase separation of the PNIPAAm brushes. As an alternative to determining the morphology of the PNIPAAm brushes during thermal treatment, incubating the PNIPAAm brushes in a poor solvent (e.g., toluene) could be used as a control experiment. ${ }^{24}$ To compare the morphologies of the PNIPAAm-grafted 2DPCG at 25 and $40{ }^{\circ} \mathrm{C}$, the samples were subjected to incubation overnight in water and toluene, respectively. Sequentially, the subjects were treated at $-40{ }^{\circ} \mathrm{C}$ 


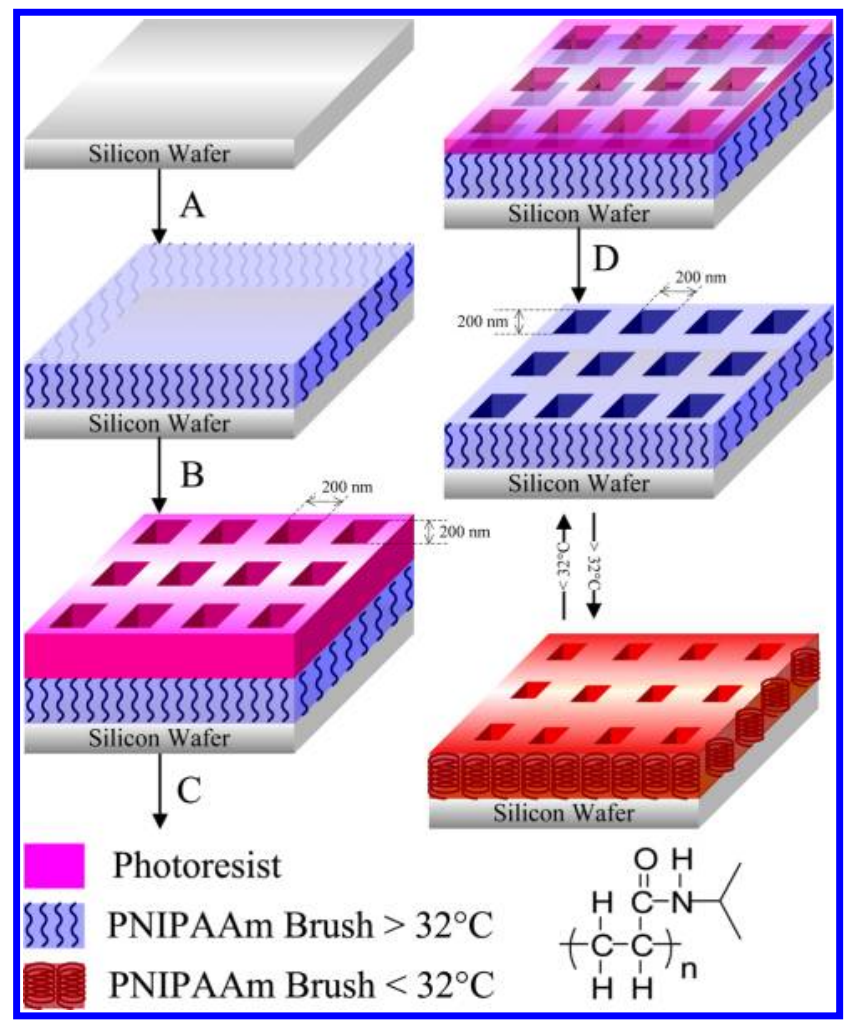

Figure 2. Schematic representation of the process used to fabricate 2DPCGs of tethered PNIPAAm. (A) Grafting of NIPAAm through surface-initiated ATRP onto a $\mathrm{Si}$ wafer from the functionalized surfaces of the initiator. (B) Photoresist spin-coated onto the Si surface presenting PNIPAAm brushes; e-beam lithography used to pattern the photoresist with hole arrays (resolution $200 \mathrm{~nm}$ ) on the surface. (C) RIE used to transfer the pattern of the hole array from the photoresist to the tethered PNIPAAm layer. (D) Photoresist removed through treatment with solvent. (E) 2DPCG of tethered PNIPAAm thin film on the surface exhibited temperature-dependent morphological and optical properties over the temperature range from 25 to $40^{\circ} \mathrm{C}$.

for $10 \mathrm{~min}$ to remove the solvent in a vacuum freeze-dryer (BENCHTOP 2K; VIRTIS; America).

Optical Properties of the Thermoresponsive 2DPCG. To readily fabricate artificial dielectric elements, one must be able to relate the effective index to the grating profile in a simple way. Many researchers have solved Maxwell's equations with relevant boundary conditions to obtain the effective indices of one-dimensional (1D) and two-dimensional (2D) subwavelength gratings. ${ }^{25}$ Alternatively, the use of effective medium theory generally yields a good approximation if the period-to-wavelength ratio is sufficiently small. For 1D gratings, the theory provides the following simple expressions for the ordinary and extraordinary indices:

$$
\begin{aligned}
& n_{\mathrm{TE}}{ }^{2}=f_{1} n_{1}{ }^{2}+f_{2} n_{2}{ }^{2} \\
& n_{\mathrm{TM}}{ }^{-2}=f_{1} n_{1}{ }^{-2}+f_{2} n_{2}{ }^{-2}
\end{aligned}
$$

where $f_{1}$ is the fill factor for medium 1 and $f_{2}$ (equal to $1-f_{1}$ ) is that for medium 2 . In the case of a $1 \mathrm{D}$ binary relief grating, the effective refractive indices for TE and TM polarizations are $n_{\mathrm{TE}}$ and $n_{\mathrm{TM}}$, respectively. The different indices for the two polarization states indicate that $1 \mathrm{D}$ subwavelength gratings exhibit form birefringence. Equations 1 and 2 have been compared frequently through rigorous calculations. ${ }^{8}$ The index in the direction normal to the grating vectors of a $2 \mathrm{D}$ subwavelength grating is given in effective-medium theory by

$$
n_{\mathrm{eff}}=\left[\left(1-f_{x} f_{y}\right) n_{1}^{2}+f_{x} f_{y} n_{2}^{2}\right]^{1 / 2}
$$

where $f_{x}$ and $f_{y}$ are the fill factors of medium 2 in the $x$ and $y$ directions, respectively (Figure 1). Although there are no simple closed-form zero-order expressions for the transverse principal effective indices of a 2D subwavelength grating, the indices can be bounded approximately through use of simple expressions derived from effective medium theory. ${ }^{26}$ For simplicity, let us consider rectangular cylindrical geometries only. To set the upper bound, the $2 \mathrm{D}$ rectangular grating can be viewed as a $1 \mathrm{D}$ grating consisting of strips aligned perpendicular to the electric field. Each strip in turn can be considered as a section of a $1 \mathrm{D}$ grating with ridges parallel to the electric field. The effective index of each strip is calculated using eq 1. These strip indices can then be employed to calculate the overall effective index, using eq 2 . This effective index is an upper bound that is exact in the static case; it is given by

$$
n_{\text {eff,upper }}=\left[\frac{1-f_{x}}{n_{1}^{2}}+\frac{f_{x}}{f_{y} n_{2}^{2}+\left(1-f_{y}\right) n_{1}^{2}}\right]^{-1 / 2}
$$

To obtain the lower bound, the $2 \mathrm{D}$ grating can be viewed as a $1 \mathrm{D}$ grating consisting of strips parallel to the electric field. The effective index of each strip is now found using eq 2 , with the overall effective index, representing the lower bound, obtained using eq 1 , giving

$$
n_{\text {eff,lower }}=\left[\left(1-f_{y}\right) n_{1}^{2}+\frac{f_{y}}{f_{x} n_{2}^{-2}+\left(1-f_{x}\right) n_{1}^{-2}}\right]^{-1 / 2}
$$

The effective-index bounds in the $y$ direction are obtained by interchanging the $x$ and $y$ subscripts. At all times, the bounds lie between the indices of the two components of the artificial dielectric. The effective refractive indices of thermoresponsive PNIPAAm

\begin{tabular}{|c|c|c|c|c|c|c|c|c|c|c|c|c|}
\hline \multirow[b]{2}{*}{$\begin{array}{l}\text { grafting time } \\
\text { (h) }\end{array}$} & \multirow[b]{2}{*}{$\begin{array}{l}\text { thickness } \\
(\mathrm{nm})\end{array}$} & \multirow[b]{2}{*}{$\begin{array}{c}M_{\mathrm{n}} \\
(\mathrm{kg} / \mathrm{mol})\end{array}$} & \multirow[b]{2}{*}{$\begin{array}{c}M_{\mathrm{w}} \\
(\mathrm{kg} / \mathrm{mol})\end{array}$} & \multirow[b]{2}{*}{$\begin{array}{c}\text { PDI }\left(M_{\mathrm{w}} /\right. \\
\left.M_{\mathrm{n}}\right)\end{array}$} & \multirow[b]{2}{*}{$\begin{array}{c}\mathrm{Sc} \\
\left(\mathrm{mg} / \mathrm{m}^{2}\right)\end{array}$} & \multirow[b]{2}{*}{$\begin{array}{c}d_{\mathrm{g}} \\
(\mathrm{nm})\end{array}$} & \multirow[b]{2}{*}{$\begin{array}{c}\sigma \\
\left(\mathrm{nm}^{-2}\right)\end{array}$} & \multicolumn{2}{|c|}{ refractive index } & \multirow[b]{2}{*}{$\begin{array}{l}\text { roughness } \\
\text { (nm) }\end{array}$} & \multicolumn{2}{|c|}{ SWCA (deg) } \\
\hline & & & & & & & & $25{ }^{\circ} \mathrm{C}$ & $40{ }^{\circ} \mathrm{C}$ & & $25^{\circ} \mathrm{C}$ & $40{ }^{\circ} \mathrm{C}$ \\
\hline 4 & 56.8 & 23.1 & 22.7 & 1.255 & 26.4 & 1.20 & 0.69 & 1.388 & 1.437 & 3.1 & $32 \pm 3$ & $80 \pm 3$ \\
\hline 8 & 90.1 & 46.2 & 58.4 & 1.264 & 55.2 & 1.18 & 0.72 & 1.390 & 1.441 & 2.9 & $31 \pm 3$ & $80 \pm 3$ \\
\hline 12 & 122.7 & 67.7 & 82.4 & 1.217 & 70.6 & 1.26 & 0.63 & 1.388 & 1.442 & 2.6 & $34 \pm 3$ & $80 \pm 3$ \\
\hline 16 & 137.4 & 72 & 86.4 & 1.2 & 79.6 & 1.23 & 0.67 & 1.391 & 1.434 & 2.3 & $35 \pm 3$ & $82 \pm 3$ \\
\hline 20 & 146.4 & 84.8 & 115.6 & 1.363 & 92.5 & 1.23 & 0.66 & 1.386 & 1.436 & 1.7 & $33 \pm 3$ & $79 \pm 3$ \\
\hline 24 & 162.2 & 94.0 & 122.2 & 1.3 & 94.2 & 1.29 & 0.60 & 1.388 & 1.438 & 1.1 & $35 \pm 3$ & $80 \pm 3$ \\
\hline
\end{tabular}
surfaces were obtained using ellipsometry at $50 \%$ relative humidity.

Table 1. Dry Layer Parameters (Molecular Weights, $M_{\mathrm{n}}$ and $M_{\mathrm{w}}$; Surface Conversion, Sc; Grafting Distance, $d_{\mathrm{g}}$; Grafting Density $\sigma$; Roughness), Refractive Indices, and Static Water Contact Angles (SWCAs) at 25 and $40{ }^{\circ} \mathrm{C}$ of Typical PNIPAAm Brush Films ${ }^{a}$ on Si Surfaces

${ }^{a} \mathrm{~A}$ bulk density for PNIPAAm of $1.07 \mathrm{~g} / \mathrm{cm}^{3}$ was used for the calculations. ${ }^{27}$ 


\section{RESULTS AND DISCUSSION}

Characterization of Tethered PNIPAAm on Si Surfaces. In a previous study, we used XPS to determine the chemical compositions of the pristine Si surface, the Si surfaces at various stages during the surface modification process, and the grafted PNIPAAm brushes. ${ }^{27}$ In the following discussion, we report the properties of thin PNIPAAm brush films in the dry state. At first, we used AFM and ellipsometry to characterize the PNIPAAm brush films in the dry state to determine their roughness, thickness, grafting density, and surface coverage of the dry film. Subsequently, we performed static water contact angle (SWCA) measurements to analyze the temperature-sensitive swelling behavior of these PNIPAAm films in contact with aqueous solutions. We determined the typical structural brush parameters-the grafting density $(\sigma)$, the distance between grafting sites $\left(d_{\mathrm{g}}\right)$, and the surface coverage $(\mathrm{Sc})$-based on the ellipsometric dry layer thickness $(h)^{28}$ and the density of bulk PNIPAAm taken from the literature. $^{29}$

$$
\begin{aligned}
& \mathrm{Sc}=\rho h \\
& d_{\mathrm{g}}=\sqrt{\frac{M_{\mathrm{n}}}{N_{\mathrm{A}} \rho h}} \\
& \sigma=d_{\mathrm{g}}{ }^{-2}
\end{aligned}
$$

Table 1 presents the calculated data for the highest grafting density achieved for each molecular weight. The similar values of $\sigma$ and $d_{\mathrm{g}}$ obtained after ATRP for various times indicate that the PNIPAAm brushes were grafted stably onto the surfaces. These systems exhibited thermoresponsiveness after modification with PNIPAAm brushes for a grafting time of $24 \mathrm{~h}$, with SWCAs of $37^{\circ}$ at $25^{\circ} \mathrm{C}$ and $80^{\circ}$ at $40^{\circ} \mathrm{C}$. The refractive indices of the tethered PNIPAAm, measured through ellipsometry at $633 \mathrm{~nm}$, also revealed thermoresponsive behavior, with refractive indices of 1.388 at $25{ }^{\circ} \mathrm{C}$ and 1.438 at $40{ }^{\circ} \mathrm{C}$. The strong temperature-dependence of both the surface wettability and refractive index further confirmed the presence of PNIPAAm layers on the surfaces. To generate thermoresponsive 2DPCGs with various hole depths, we used systems that had been grafted with PNIPAAm for $24 \mathrm{~h}$.

Morphologies of Thermoresponsive 2DPCGs of Tethered PNIPAAm. In the etching process, we used photolithography to treat the PNIPAAm brushes covered with a patterned photoresist of hole arrays. According to the effective-medium theory, the fill factors in the $x\left(f_{x}\right)$ and $y\left(f_{y}\right)$ directions represent the scales of the square hole structures (Figure 1). We varied the hole depths of the 2DPCGs by etching the tethered PNIPAAm for 60, 120, and $180 \mathrm{~s}-$ providing samples denoted herein as HA1, HA2, and HA3, respectively-and then used AFM to visualize the morphologies of the PNIPAAm brushes in the 2DPCGs. Figure 3a displays 2D, 3D, and line cross-section analysis AFM topographic images of $\mathrm{HA} 2$, revealing that the PNIPAAm brushes on the Si surface existed at $25^{\circ} \mathrm{C}$ as a dense distinctive overlayer, with a hole scale of approximately $200 \mathrm{~nm}$, within a scanning area of $10 \mu \mathrm{m} \times 10 \mu \mathrm{m}$. The line cross-section analysis clearly revealed the texture of the PNIPAAm brushes at $50 \%$ relative humidity. Each hole of the 2DPCG was fabricated successfully with a top scale of $203 \pm 8 \mathrm{~nm}$ and a bottom scale of $157 \pm 6 \mathrm{~nm}$ in the tethered PNIPAAm layer, with a similar depth $(112 \pm 5 \mathrm{~nm})$ and scale. A brief rinse (ca. 15 s) in water

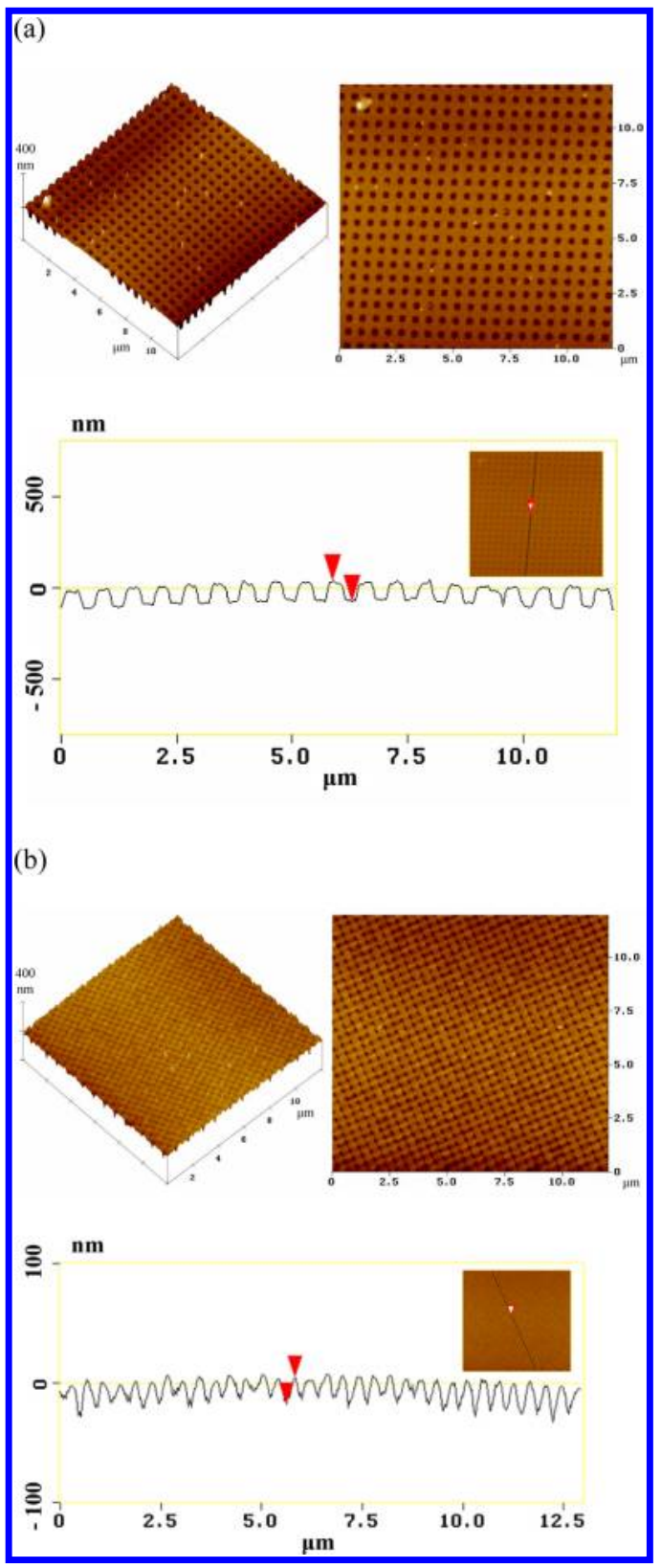

Figure 3. $2 \mathrm{D}$ (left), 3D (right), and line cross-section (bottom) analysis AFM topographic images of the surfaces of 200-nm-resolution 2DPCGs of PNIPAAm brushes, fabricated through an etching process, at (a) 25 and (b) $40{ }^{\circ} \mathrm{C}$.

at $40{ }^{\circ} \mathrm{C}$ and subsequent lyophilization led to a conformational change as a result of deswelling and phase-separation of the PNIPAAm brushes. Accordingly, the regular hole array of PNIPAAm brushes was erased after heating the surface at 40 ${ }^{\circ} \mathrm{C}$, resulting in the appearance of a rough surface (Figure $3 \mathrm{~b}$ ). Reimmersion in water at $25{ }^{\circ} \mathrm{C}$ and subsequent lyophilization resulted in rehydration of the PNIPAAm and extension of the film to a swelled state. The nonapparent hole array of PNIPAAm brushes at $40{ }^{\circ} \mathrm{C}$ returned to a regular hole array when we decreased the temperature from 40 to $25{ }^{\circ} \mathrm{C}$, confirming the reversible temperature-responsiveness of the 2DPCG; nanohole features having an apparent depth of $112 \pm$ $6 \mathrm{~nm}$ were observed only for HA2. ${ }^{30}$ This behavior was reversible over five cycles. Both thermal treatment and treatment with a poor solvent led to phase separation of the 
PNIPAAm brushes. When we incubated the patterned PNIPAAm brushes in toluene overnight and then analyzed them using AFM, we observed a surface morphology similar to that in Figure $3 b$; hence, we conclude that the erasing of the hole array in Figure $3 \mathrm{~b}$ was truly a result of temperatureinduced structural changes, allowing such systems to be used for temperature-dependent switching. Furthermore, we also compared the scales of the hole arrays at $40{ }^{\circ} \mathrm{C}$ in relation to the original depths etched for HA1 and HA3. Our 200-nmresolution 2DPCGs of PNIPAAm brushes HA1, HA2, and HA3 exhibited various depths and scales after five cycles of thermal switching (Figure 4). The scales of the 2DPCGs in the

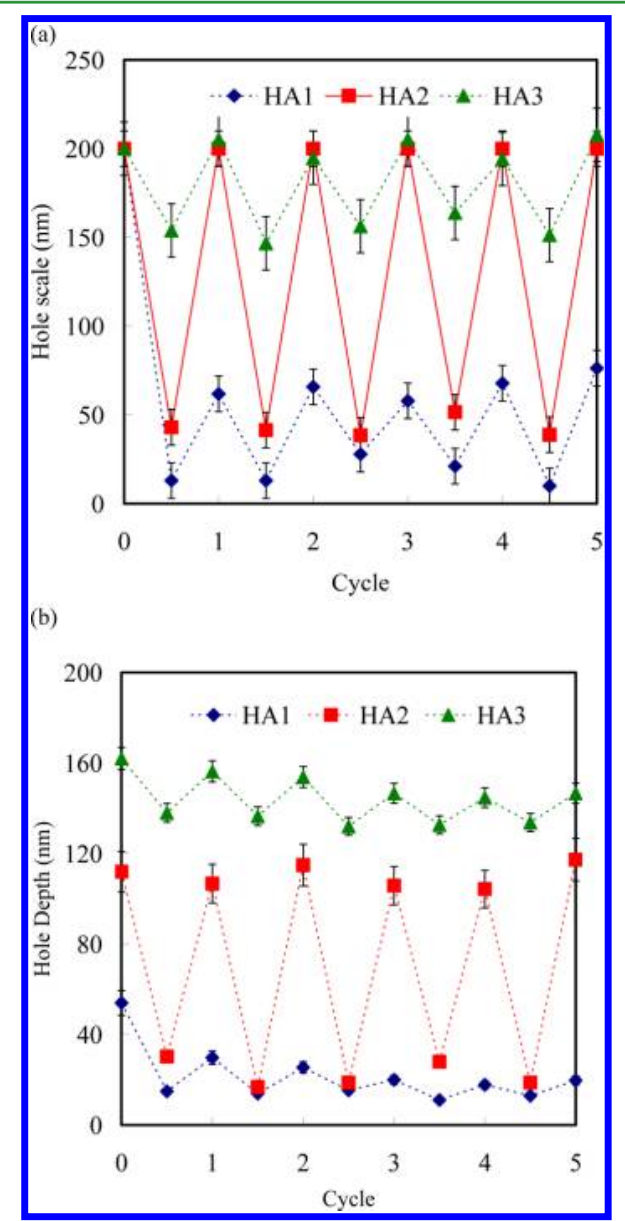

Figure 4. Variations in the (a) scale and (b) depth of 2DPCG surfaces of tethered PNIPAAm (HA1, HA2, and HA3) upon five cycles of switching of the temperature between 25 and $40{ }^{\circ} \mathrm{C}$.

initial states matched the original pattern scale $(200 \mathrm{~nm})$ of the photoresist. The hole scale and depth for HA1 did not, however, return totally to the original nanoscale after the first cycle of thermal treatment for HA1 (Figure 4). In addition, the scale and depth of the holes in HA3 decreased slightly in the phase-separated state of PNIPAAm but retained the porous surface. The pattern of the shallower 2DPCG HA1 disappeared after thermal treatment because its holes were nearly filled completely with the rearranged residual polymer segments. For the deeper 2DPCG HA3, the rearranged residual polymer segments could not completely fill the hole pattern after thermal treatment. Therefore, the shallower and deeper 2DPCG did not undergo significant changes in morphology after each temperature switching cycle. Both of scale and depth for HA2 exhibited the most efficient thermal responses. Thus, the efficiency of the thermal responsiveness of the porous surface was dependent on the initial conditions in terms of the hole depth.

Temperature-Switchable Optical Properties of 2DPCGs of Tethered PNIPAAm. Figure 5a displays the thickness and refractive index of a flat surface of tethered

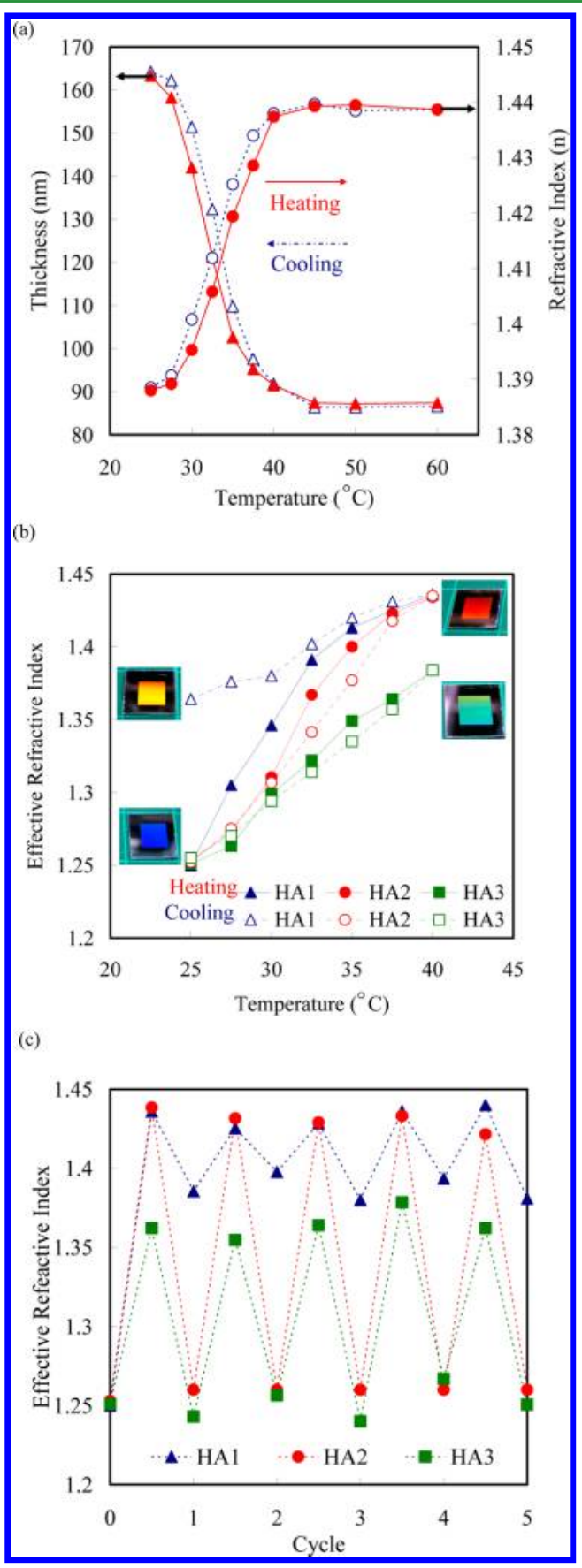

Figure 5. (a) Thermal responses of the thicknesses and refractive indices of PNIPAAm brushes as a function of temperature. (b and c) Values of $n_{\text {eff }}$ of 2DPCGs of PNIPAAm brushes (HA1, HA2, and HA3), measured using ellipsometry, (b) plotted as a function of temperature and (c) after five cycles of tuning of the temperature between 25 and $40{ }^{\circ} \mathrm{C}$. Photographic images in part b demonstrate the grating effects of the surfaces of the 2DPCGs HA1, HA2, and HA3 at 25 and $40{ }^{\circ} \mathrm{C}$. 
PNIPAAm as a function of temperature, as determined using environmental ellipsometry at a wavelength of $633 \mathrm{~nm}$; the data are consistent with those from a previous report. ${ }^{31}$ The lower critical solution temperature (LCST) transition appears to occur over the broad temperature range $25-35^{\circ} \mathrm{C}$, with the majority of the transition between 28 and $40{ }^{\circ} \mathrm{C}$. We used ellipsometry to probe the thermal response of a PNIPAAm brush. Figure $5 \mathrm{~b}$ presents the effective refractive indices $\left(n_{\text {eff }}\right)$ of the 2DPCGs HA1, HA2, and HA3 plotted with respect to temperature. The values of $n_{\text {eff }}$ of the 2DPCG were related substantially to the degree of phase separation of the PNIPAAm and the concomitant degree of collapse of the polymer brushes. Moreover, the 2DPCGs HA1, HA2, and HA3 exhibited values of $n_{\text {eff }}$ in their initial states that were less than that of a flat PNIPAAm brush, indicating that the value of $n_{\text {eff }}$ is related to the hole depth. Figure $5 \mathrm{~b}$ reveals that the value of $n_{\text {eff }}$ decreased with respect to the hole depth of the 2DPCG, due to the increase in the filling factor of air inside the tethered PNIPAAm $\left(f_{\text {air }}\right)$. After increasing the temperature from 25 to $40{ }^{\circ} \mathrm{C}$, the value of $n_{\text {eff }}$ for HA1 increased slightly, reaching close to that of a flat PNIPAAm brush. The values of $n_{\text {eff }}$ after performing a heating and cooling cycle did not return completely to the original value. For HA2, the value of $n_{\text {eff }}$ increased abruptly from 1.228 to 1.435 upon heating from 25 to $40{ }^{\circ} \mathrm{C}$; it returned to 1.227 after cooling from 40 to $25{ }^{\circ} \mathrm{C}$. As expected, the value of $n_{\text {eff }}$ for HA2 could be alternated reversibly between 1.228 and 1.435 through heating and cooling processes, due to the variation of $f_{\text {air }}$. In addition, the value of $n_{\text {eff }}$ for HA3 could be also alternated reversibly between 1.131 and 1.246 as a result of a slight variation in the value of $f_{\text {air }}$. Figure $5 \mathrm{~b}$ presents photographic images of the 2DPCGs of PNIPAAm brushes at 25 and $40{ }^{\circ} \mathrm{C}$, obtained at an angle $\beta$ of approximately $10-20^{\circ}$ (Figure 1 ). These 2 DPCG samples were prepared into a die using an etching process with a $1 \mathrm{~cm} \times 1 \mathrm{~cm}$ contact mask. The light impinging the sample at an incident angle was linearly polarized, with the electric field vibration parallel $(p)$ or perpendicular $(s)$ to the plane of incidence; the reflected light was also $p$ - or $s$-polarized. In this study, we observed by the naked eye the reflection of the 2DPCGs of tethered PNIPAAm under an invariable angle $\beta .{ }^{32}$ At values of $\beta$ of $10-20^{\circ}$, the 2 DPCGs HA1 and HA2 displayed blue and red colors at 25 and $40{ }^{\circ} \mathrm{C}$, respectively. For HA1, however, the red color returned to yellow, instead of the initial blue, after decreasing the temperature from 40 to $25{ }^{\circ} \mathrm{C}$. For HA3, the color changed from blue to green during the thermal switching cycles. For the 2DPCGs of PNIPAAm brushes, this behavior was reversible for at least five cycles (Figure 5c). The effect of diffractive grating is dependent on the period and/or the angle of observation when the structure scale of a grating is larger than wavelength. Therefore, the period and angle of observation are fixed possibly in our case. Furthermore, the scale of each pillar of 2DPRG is $200 \mathrm{~nm}$ less than wavelength; it may not follow the diffraction grating theory that filling factor does not affect the color change of the grating. The pure color display of 2DPRG indicates that the effect of photonics may be predominant for the SWS 2DPRG and change with filling factor. Furthermore, the resultant 2DPCGs were capable of undergoing reversible, thermally responsive structural changes. A 2DPCG can be regarded as a layer that is filled with air; in eq $3, n_{1}$ and $n_{2}$ represent the refractive indices of the tethered PNIPAAm layer and air, respectively. We calculated the values of $f_{\text {air }}$ in the $x$ direction $\left(f_{\text {air }, x}\right)$ by using the measured values of $n_{\text {eff }}$ and $f_{\text {air }}$ in the $y$ direction $\left(f_{\text {air }, y}\right)$, itself determined from the changes in hole scale during the heating and cooling processes. For the convenience, the fill factor in the $x$ direction $\left(f_{\text {air }, x}\right)$ is set equal to that in the $y$ direction $\left(f_{\text {air }, y}\right)$ for a description of the hole structure with diameter and depth. Equations 3-5 could be rewritten as $6-8$ in the following, respectively.

$$
\begin{aligned}
& n_{\text {eff }}=\left[\left(1-f^{2}\right) n_{1}^{2}+f^{2} n_{2}^{2}\right]^{1 / 2} \\
& n_{\text {eff,upper }}=\left[\frac{1-f}{n_{1}^{2}}+\frac{f}{f n_{2}^{2}+(1-f) n_{1}^{2}}\right]^{-1 / 2} \\
& n_{\text {eff,lower }}=\left[(1-f) n_{1}^{2}+\frac{f}{f n_{2}^{-2}+(1-f) n_{1}^{-2}}\right]^{-1 / 2}
\end{aligned}
$$

The effective medium theory certainly obtains much more accurate results only when the period is sufficiently smaller than wavelength. Therefore, we use the bounded indices expressions to fit our results. The report suggests that the effective indices are on the average increased by at most $3 \%$ for a $2 \mathrm{D} 400-\mathrm{nm}$ period symmetric grating at a wavelength of $633 \mathrm{~nm} .{ }^{33}$ The inaccuracy of refractive indices for $200-\mathrm{nm}$ scale 2 DPRG at a wavelength of $633 \mathrm{~nm}$ may be on the average less than $3 \%$. Figure 6 presents the temperature-dependence of the calculated

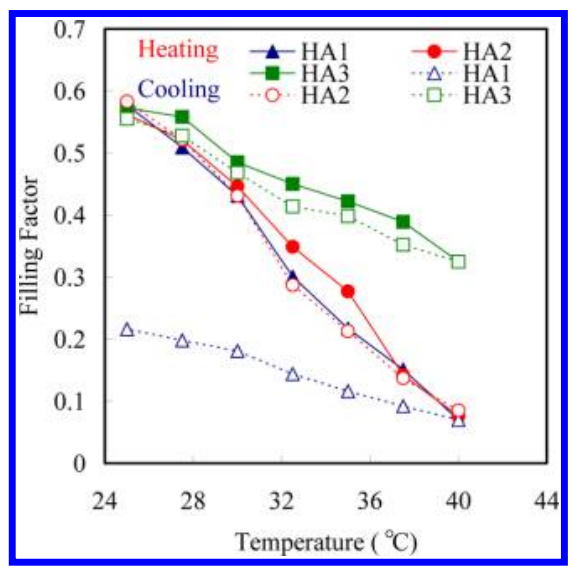

Figure 6. Changes in the values of $f_{\text {air }, x}$ of the 2DPCGs of tethered PNIPAAm plotted with respect to the temperature from 25 to $40{ }^{\circ} \mathrm{C}$.

values of $f$ of the 2DPCGs HA1, HA2, and HA3. The results suggest that the 2DPCGs underwent a decrease in the filling volume of air inside the PNIPAAm film upon increasing the temperature. The values of $f$ for HA2 exhibited the greatest efficiency of temperature-dependence over the temperature range from 25 to $40{ }^{\circ} \mathrm{C}$, consistent with a major structural change. Moreover, we used the refractive indices of a PNIPAAm thin film at $633 \mathrm{~nm}$ at 25 to $40{ }^{\circ} \mathrm{C}$-measured as 1.388 and 1.438 , respectively-as standard values in eqs 7 and 8 to compare the measured and calculated values of $n_{\text {eff }}$ and $f$ of these 2DPCGs. We constructed the model for the values of $n_{\text {eff }}$ and $f$ at 25 and $40{ }^{\circ} \mathrm{C}$, described by eqs 7 and 8 , to fit data only at $633 \mathrm{~nm}$ to avoid the lower wavelength regions that are strongly absorbed by the surface coverslip; Figures $7 \mathrm{a}-\mathrm{c}$ present the data for HA1, HA2, and HA3, respectively. Because we used solely refractive indices of 1.388 and 1.438 at 25 and $40{ }^{\circ} \mathrm{C}$, respectively, as standard values, the measured values of $n_{\text {eff }}$ located within the region bounded by eqs 7 and 8 at 25 and $40{ }^{\circ} \mathrm{C}$ could be regarded as a close fit. Indeed, the model data perfectly matched the measured data for HA1, HA2, and HA3 during thermal treatment. In addition, the observed values of 


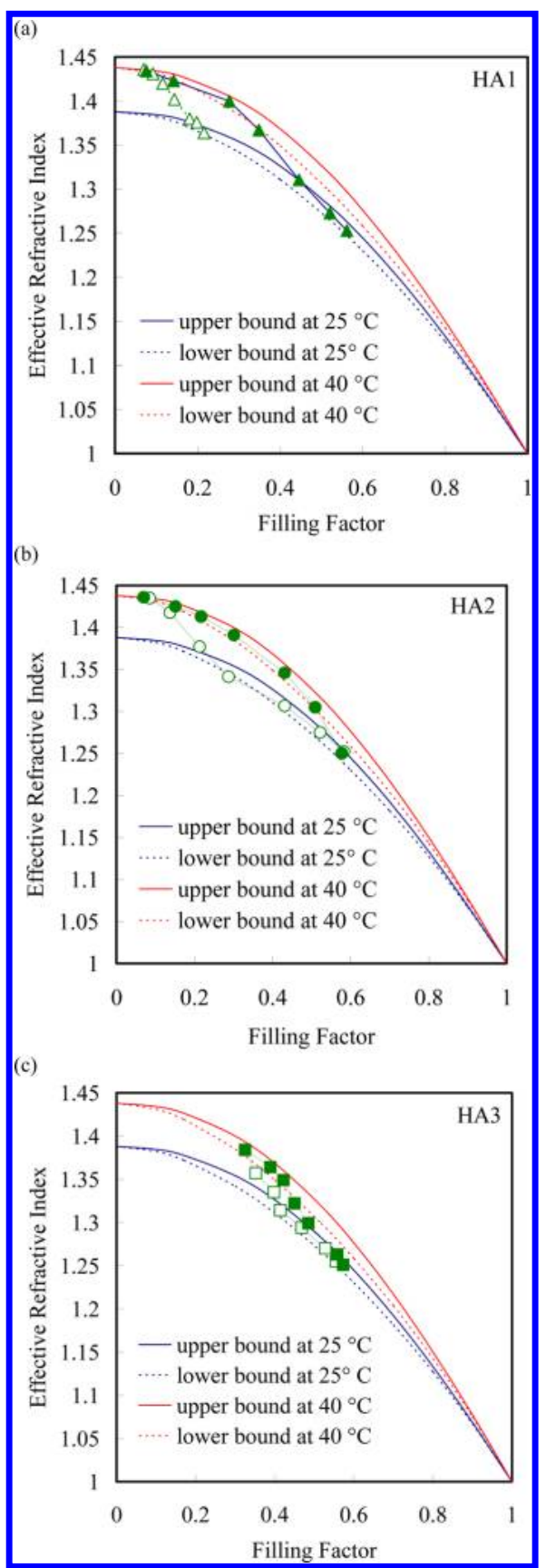

Figure 7. Values of $n_{\text {eff }}$ of 2DPCGs of PNIPAAm brushes plotted with respect to the values of $f_{\text {air }, x}$. The plots were fitted to eqs 4 and 5 at 25 and $40{ }^{\circ} \mathrm{C}$ for (a) HA1, (b) HA2, and (c) HA3.

$n_{\text {eff }}$ and $f$ for HAl were totally reversible upon cooling with hysteresis, indicating a phase transition involving rearrangement of the pattern; the values of $n_{\text {eff }}$ and $f$ did not, however, return to their initial values after decreasing the temperature from 40 to $25{ }^{\circ} \mathrm{C}$, revealing an open hysteresis loop (Figure 7a). The value of $f$ for HA2 was approximate zero, indicating that the tethered PNIPAAm layer without pores featured a flat surface; Figure $7 \mathrm{~b}$ reveals a closed hysteresis loop with approximately parallel branches for HA2. For HA3, the hysteresis loop was not obvious, indicating that the hole array of this structure remained principally in its initial state (Figure 7c). The results suggest that 2DPCGs of PNIPAAm brush layers possessing a hole depth of less than $112 \mathrm{~nm}$ recover, presumably to a flat surface, after thermal treatment, leading to less-steep responses of the values of $n_{\text {eff }}$ and $f$ to the thermal treatment, such that the hysteresis loops become somewhat broader.

ARS surfaces have many advantages over coatings with subwavelength microstructures. ${ }^{34,35}$ For example, the gradual change in refractive index decreases the reflection of light at the substrate surface. ${ }^{36}$ Nanoporous structures suitable for use as ARS coating sensors may be created and erased during thermal tuning cycles. 2DPCGs of PNIPAAm brushes without TM or TE polarization can be regarded as porous surfaces. The morphology of our 2DPCG HA2 alternated between a hole array and a rough structure as a result of reversible swelling transitions. Reflectivity data of Si wafer substrates fabricated on one side with these 2DPCG films revealed that the hole arrays effectively suppressed reflective losses near the quarterwavelength optical thickness $(633 \mathrm{~nm})$, reaching as low as $13.7 \%$ at $25{ }^{\circ} \mathrm{C}$ for HA2 (Figure 8a). In the nonporous state

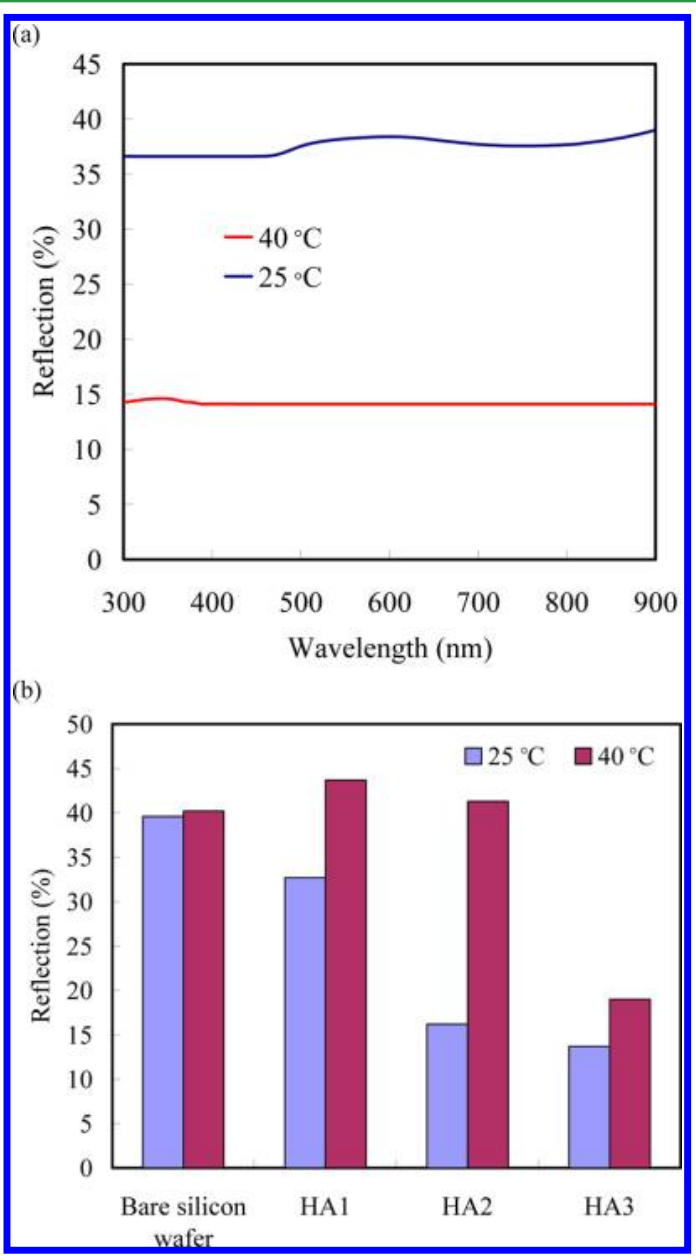

Figure 8. (a) Plot of near-normal reflection with respect to wavelength for a Si substrate coated on one side with HA2. (b) Near-normal reflections of a bare Si wafer, HA1, HA2, and HA3 at $633 \mathrm{~nm}$.

(rough structure), this thin film no longer behaved as an antireflection coating, resulting in a reflective loss (ca. 39.6\%) mostly from the Si substrate. The PNIPAAm grafted onto the Si wafer without a hole array underwent a slight decreasing in reflection to approximately $37.6 \%$ at $633 \mathrm{~nm}$, due to the increased roughness on the polished surface (Figure $8 \mathrm{~b}$ ). As expected, the reflection of the phase-separated 2DPCG HA2 alternated from approximately $36.6 \%$ to approximately $14.4 \%$ as a result of the reversible swelling transition. 


\section{CONCLUSIONS}

We have used the "grafting from" system with ATRP to prepare densely grafted PNIPAAm brushes on the surface of Si wafers and then used an etching process to generate 200-nm-scale hole arrays for use as 2DPCGs. By selecting a suitably functionalized polymer and optimizing the patterning process, we could readily alter the depths and profiles of the nanostructures. Light impinging the 2DPCGs of tethered PNIPAAm having a suitable etching depth at an incident angle of $70-80^{\circ}$ appeared as pure blue and red colors, observable by the naked eye, at temperatures below and above the LCST, respectively. Such 2DPCGs of tethered PNIPAAm can be fabricated over a large area with the capability to detect variations in temperature. In addition, continuous 2DPCGs of tethered PNIPAAm provide high antireflection performance because they undergo conformational changes that result in a changes in effective refractive index in the modified grating region. Such controllable and reversible gratings prepared from tunable films are ideal materials for optical technologies, such as the antireflection coatings and tunable 2DPCGs.

\section{AUTHOR INFORMATION}

\section{Corresponding Author}

*Tel.: +886-2-27376523. Fax: +886-2-27376544. E-mail: jkchen@mail.ntust.edu.tw.

\section{Notes}

The authors declare no competing financial interest.

\section{ACKNOWLEDGMENTS}

We thank the National Science Council of the Republic of China for supporting this research financially and the National Nano Device Laboratory for assistance with the electron beam lithography and RIE, respectively.

\section{REFERENCES}

(1) Ono, Y.; Kimura, Y.; Ohta, Y.; Nishida, N. Appl. Opt. 1987, 26, $1142-1146$.

(2) Stoykovich, M. P.; Müller, M.; Kim, S. O.; Solak, H. H.; Edwards, E. W.; de Pablo, J. J.; Nealey, P. F. Science 2005, 308, 1442-1446.

(3) Brolo, A. G. Nat. Photonics 2012, 6, 709-713.

(4) Chang, C. H.; Dominguez-Gaballero, J. A.; Choi, H. J.; Barbastathis, G. Opt. Lett. 2011, 36, 2354-2356.

(5) Min, W.-L.; Jiang, P.; Jiang, B. Nanotechnology 2008, 19 (47), 475604.

(6) Chen, J. K.; Bai, B. J. J. Phys. Chem. C 2011, 115, 21341-21350.

(7) Koo, H. Y.; Yi, D. K.; Yoo, S. J.; Kim, D. Y. Adv. Mater. 2004, 16

(3), 274-277.

(8) Raguin, D. H.; Morris, G. M. Appl. Opt. 1993, 32, 1154-1167.

(9) Raguin, D. H.; Morris, G. M. Appl. Opt. 1993, 32, 2582-2598.

(10) Warren, M.; Smith, R.; Vawter, G.; Wendt, J. Opt. Lett. 1995, 20, 1441-1443.

(11) Uhlmann, D. R.; Suratwala, T.; Davidson, K.; Boulton, J. M.; Teowee, G. J. Non-Cryst. Solids 1997, 218, 113-122.

(12) Hattori, H. Adv. Mater. 2001, 13, 51-54.

(13) Ibn-Elhaj, M.; Schadt, M. Nature 2001, 410, 796-799.

(14) Chen, J.-K.; Chen, Z.-Y.; Lin, H.-C.; Hong, P.-D.; Chang, F.-C. ACS Appl. Mater. Interfaces 2009, 1, 1525-1532.

(15) Peppas, N. A.; Hilt, J. Z.; Khademhosseini, A.; Langer, R. Adv. Mater. 2006, 18, 1345-1360.

(16) Morsch, S.; Schofield, W. C. E.; Badyal, J. P. S. Langmuir 2010, 26, 12342-12350.

(17) Hu, Z.; Chen, Y.; Wang, C.; Zheng, Y.; Li, Y. Nature 1998, 393, 149-152.

(18) Ye, G.; Wang, X. Biosens. Bioelectron. 2010, 26, 772-777.
(19) Chen, J. K.; Zhuang, A.-L. J. Phys. Chem. C 2010, 114, 1180111809.

(20) Chen, J. K.; Wang, J.-H.; Fan, S.-K.; Chang, J.-Y. ACS Appl. Mater. Interfaces 2012, 4, 1935-1947.

(21) Shiratori, S. S.; Rubner, M. F. Macromolecules 2000, 33, 42134219.

(22) Chen, J. K.; Hsieh, C. Y.; Huang, C. F.; Li, P. M.; Kuo, S. W.; Chang, F. C. Macromolecules 2008, 41, 8729-8736.

(23) Lo, C. Y.; Huang, Y.-R.; Liao, K.-S.; Kuo, S.-A.; Wei, S.-P. Microelectron. Eng. 2011, 88, 2657-2661.

(24) Gupta, S.; Agrawal, M.; Uhlmann, P.; Simon, F.; Stamm, M. Chem. Mater. 2010, 22, 504-509.

(25) Grann, E. B.; Moharam, M. G.; Pommet, D. A. J. Opt. Soc. Am. A 1994, 11, 2695-2703.

(26) Jackson, J. L.; Coriell, S. R. J. Appl. Phys. 1968, 39, 2349.

(27) Chen, J.-K.; Li, J.-Y. Sens. Actuators B 2010, 150, 314-320.

(28) Brittain, W. J.; Minko, S. J. Polym. Sci. A: Pol. Chem. 2007, 45, 35053512

(29) Saunders, B. R. Langmuir 2004, 20, 3925-3932.

(30) Tu, H.; Heitzman, C. E.; Braun, P. V. Langmuir 2004, 20, $8313-8320$

(31) Garner, B. W.; Cai, T.; Ghosh, S.; Hu, Z.; Neogi, A. Appl. Phys. Express 2009, 2, 057001.

(32) Burton, Z.; Bhushan, B. Nano Lett. 2005, 5, 1607-1613.

(33) Chen, F. T.; Craighead, H. G. Opt. Lett. 1995, 20 (2), 121-123.

(34) Hiller, J.; Mendelsohn, J. D.; Rubner, M. F. Nat. Mater. 2002, 1, $59-63$.

(35) Clapham, P. B.; Hutley, M. C. Nature 1973, 244, 281-282.

(36) Pacholski, C.; Morhard, C.; Spatz, J. P.; Lehr, D.; Schulze, M.; Kley, E. B.; Tunnermann, A.; Helgert, M.; Sundermann, M.; Brunner, R. Appl. Opt. 2012, 51, 8-14. 